\title{
Quetiapine Dosage Across Diagnostic Categories
}

\author{
Yasser Khazaal $\cdot$ Anne Chatton · Riaz Khan $\cdot$ Daniele Zullino
}

Published online: 14 January 2009

(C) Springer Science+Business Media, LLC 2009

\begin{abstract}
Objective The aim of the current study was to evaluate quetiapine doses used across diagnosis categories in a sample of psychiatric inpatients. Methods Discharge letters of all adult inpatients who had received quetiapine between 1999 and 2005 were retrospectively reviewed. Logistic regressions were carried-out to assess links between quetiapine discharge dosage ( $\geq 800 \mathrm{mg} /$ day vs. $<800 \mathrm{mg} /$ day), diagnostic categories, substance abuse or dependence, benzodiazepine discharge doses, age and sex. Results The data of 231 patients were included. Five hundred and for discharge documents were analyzed: 113 for psychotic disorders, 190 for personality disorders, 134 for bipolar and schizoaffective bipolar disorders, 29 for unipolar depression or anxiety disorders, and 35 for mental retardation. Considering psychotic disorders as a reference group, patients with personality disorders were statistically significantly less likely to be in the high quetiapine dosage group at discharge $(P=0.007, \mathrm{OR}=0.1$ and $\mathrm{CI}[0.03 ; 0.6])$. Conclusions Quetiapine seems to be used in a variety of clinical situations, with a wide range of doses and a lower dosage in patients treated for personality disorders.
\end{abstract}

Keywords Quetiapine · Antipsychotic drugs · Bipolar disorder · Schizophrenia · Borderline personality disorder

\section{Introduction}

Quetiapine is a novel antipsychotic drug labeled for the treatment of patients with schizophrenia [1], bipolar mania [2] and bipolar depression [3].

The registration studies of quetiapine suggest a target dose of quetiapine ranging from 300 to $500 \mathrm{mg} /$ day for schizophrenia, and a maximum dosage up to $800 \mathrm{mg} /$ day [4].

Quetiapine has been tried across multiple diagnosis categories and seems to be used, among other atypical antipsychotics, in clinical practice for an expanding range of disorders [5].

Y. Khazaal $(\bowtie) \cdot$ A. Chatton · R. Khan · D. Zullino

Division of Substance Abuse, Geneva University Hospitals, Rue Verte 2, 1205 Geneva, Switzerland

e-mail: yasser.khazaal@hcuge.ch 
Several recent studies have demonstrated quetiapine's clinical benefits in patients with depression, impulsivity, aggression, and substance abuse disorders [6-10].

Preliminary works suggest interest of quetiapine in monotherapy or augmentation strategy for a wide range of other psychiatric disorders such as antisocial personality disorder (600-800 mg/day) [11], borderline personality disorders (175-400 mg/day) in one study [12] and at higher dosage in a recent one (400-800 $\mathrm{mg} /$ day, with average daily dosage: $540 \mathrm{mg} /$ day) [13], resistant depression (mean dosage: $166.67 \pm 211.69 \mathrm{mg} /$ day) [14], post traumatic stress disorders [15] (mean dosage: $216 \mathrm{mg} /$ day), resistant obsessive compulsive disorders (quetiapine dosages titrated upward to $300 \mathrm{mg} / \mathrm{day}$, mean dose: $215 \pm 124 \mathrm{mg} /$ day) [16]. Controversial data, for example, on the lack of efficacy of quetiapine at dosage lower than $150 \mathrm{mg}$ /day highlights the importance of a precise determination of the potentially effective dosage [17].

Whereas most validation studies on the efficacy of quetiapine used up to a maximum dosage of $800 \mathrm{mg} / \mathrm{day}$, naturalistic reports show a current use in higher dosage in a variety of clinical situations $[4,18,19]$. Due to this gap between validation studies (quetiapine used up to $800 \mathrm{mg} /$ day) and naturalistic reports (quetiapine frequently used at higher doses than $800 \mathrm{mg} /$ day), the aim of the current retrospective study was to evaluate prescription of quetiapine in dosage $<800 \mathrm{mg} /$ day and $\geq 800 \mathrm{mg} /$ day across diagnostic categories in a naturalistic sample of psychiatric inpatients.

\section{Methods}

All patients described in this retrospective cross-sectional descriptive study had been treated in the Psychiatric hospital of the University Hospital of Vaud, Lausanne, Switzerland. Discharge letters of all adult inpatients who had received a prescription for quetiapine during at least one hospitalization (during the stay in the hospital and/or at discharge) between December 1999 (introduction of quetiapine in Switzerland) and September 2005 were retrospectively reviewed. Discharge letters are the official and final medical document, signed by a resident in psychiatry and a senior psychiatrist, which summarize events, diagnosis, symptoms evolution and treatment during hospitalization and contains systematic reporting of all treatments at discharge. Discharge letters containing the word quetiapine were identified through a computerized research. Letters that did not contain this word were not checked. Letters in which quetiapine was not reported at discharge were excluded from the analysis.

The following information was recorded from the discharge letter: baseline demographic characteristics, psychiatric diagnostic including active substance abuse or dependence, number of previous hospitalization, medication including benzodiazepine dosage at discharge (in lorazepam equivalent), quetiapine dose at discharge, duration of hospitalization, civil compulsory hospitalizations. Diagnoses were established according to DSM-IV criteria by the residents in psychiatry and senior psychiatrists.

The presented data are drawn from a study protocol approved by the local Ethical committee and the institutional review board.

\section{Analyses}

Statistical analyses were carried out using SPSS for Windows (version 11.0). 
An initial exploratory analysis involved calculation of means, standard deviation and median for age and discharge quetiapine and benzodiazepine (in lorazepam equivalent) dosages.

Diagnostic categories are regrouped in 5 categories as follows:

(1) Bipolar spectrum including: bipolar disorder and schizoaffective bipolar disorder in manic or mixed states (with exclusion of bipolar depression due to small sample size: 6 discharge letters), (2) Psychotic disorders including: schizophrenia and schizoaffective disorders depressive type, psychotic disorders not otherwise specified, delusional disorders, (3) Unipolar depression and anxiety disorders, (4) Personality disorders (cluster B), (5) Mental retardation.

Schizoaffective disorder, bipolar type and bipolar disorder were in the same category "Bipolar spectrum" in accordance with previous results [19] showing that higher quetiapine dosage is rather related to the mood episode type than to the category of bipolar disorder or schizoaffective bipolar disorder.

When co-morbidity was mentioned, the categorization was considered in consideration of the diagnosis linked to the hospitalization as reported in the discharge letter. Substance abuse or dependence was considered as, a co-morbidity.

Quetiapine doses between diagnostic categories were studied in consideration of high ( $\geq 800 \mathrm{mg} /$ day) vs. low ( $<800 \mathrm{mg} /$ day) quetiapine dosage repartition, in consideration to the $800 \mathrm{mg}$ cut-off of validation studies and the frequency of prescription of doses higher than $800 \mathrm{mg} /$ day in clinical practice.

Analyses were conducted on the sample of patients, taking the mean quetiapine dose over all the hospitalizations made by each patient (the average of the different daily discharge dose prescribed for each patient). The mean quetiapine doses as the dependent variable of age, benzodiazepine discharge doses, diagnostic categories, sex, as well as substance abuse variables as predictors were tentatively analyzed through a multiple linear regression. Because of strong deviance from normality and non-constant variance, the data were finally analyzed by a logistic regression model providing a better fit. The mean quetiapine discharge dose was categorized into high dose: $\geq 800 \mathrm{mg}$ vs. low dose: $<800 \mathrm{mg} /$ day. Goodness of fit of the model was assessed through the logarithm of the likelihood and the Nagelkerke $\mathrm{R}^{2}$ statistics. Leverage values were also analyzed for detecting cases that have a large impact on the predicted values. For all analyses, a significance level of $P \leq 0.05$ was used.

\section{Results}

Five hundred and four discharge letters were analyzed after exclusion of 38 discharge letters of patients who were not discharged with quetiapine (refusal of treatment, switch, to another antipsychotic agent, quetiapine side effects, considering no reason for quetiapine maintenance treatment). The data of 231 patients [female (59\%)], were included into the analyses. During the studied period the included patients totalized 504 hospitalizations: 113 hospitalizations for psychotic episodes, 190 for personality disorders (cluster B), 137 for bipolar and schizoaffective bipolar disorder, 29 for unipolar depression [15] or anxiety disorder [14] and 35 for mental retardation. Only three patients (7 hospitalizations) received quetiapine for two different diagnoses in successive hospitalizations, the last diagnosis was considered for each of these patients because it was considered as more accurate by the clinicians. Forty-three percent of the study sample had a co-morbidity of 
substance abuse or dependence to one or more substances, most frequently alcohol (50\%), marijuana $(40 \%)$, cocaine $(15 \%)$ and opiate $(4 \%)$.

The socio-demographic and clinical characteristics of the patients and the hospitalizations are shown in Table 1 . The logistic regression performed well with an overall percentage of $85 \%$ of the cases correctly classified (high: $\geq 800 \mathrm{mg} / \mathrm{day}$ vs. low: $<800 \mathrm{mg}$ /day quetiapine dosage category). The $\mathrm{R}^{2}$ Nagelkerke statistics show that about $67 \%$ of the variation in the outcome variable is explained by the logistic regression model. The variables, age and diagnosis, are statistically significant $(P<0.0005$ and $P=0.002$ respectively) while benzodiazepine dosage, sex and substance abuse or dependence are not. Compared to those with psychotic disorders (Table 2), patients with personality disorders were significantly less likely to be in the high dose category $(P=0.007, \mathrm{OR}=0.1$ and $\mathrm{CI}[0.03 ; 0.6])$. Considering age variable, an increase of 1 year decreases the likelihood to be in the high quetiapine dose group $(P<0.0005$, $\mathrm{OR}=0.96$ and $\mathrm{CI}[0.93 ; 0.98])$.

Table 1 Sociodemographic and clinical characteristics (the reported diagnosis is the diagnosis linked to the hospitalization)

\begin{tabular}{ll}
\hline Patients $(n)$ & 231 \\
Female $(\%)$ & $59 \%$ \\
Age, years (mean \pm SD; median) & $36.3 \pm 11.3 ; 35$ \\
Numbers of previous hospitalization (mean \pm SD; median) & $7.4 \pm 11.3 ; 4$ \\
Daily mean quetiapine dosage & $393.8 \pm 320.2$ \\
Mean quetiapine dosage by episode & \\
$\quad$ (1) Psychotic disorders & $557.3 \pm 277.5$ \\
(2) Bipolar disorders and schizoaffective, bipolar type & $435 \pm 395.4$ \\
(3) Personality disorders & $251.6 \pm 211.7$ \\
$\quad$ (4) Depressive and anxiety disorders & $279.2 \pm 279.9$ \\
$\quad$ (5) Mental retardation & $414.2 \pm 223$ \\
Number of subjects by episode type $(n)$ & \\
$\quad$ (1) Psychotic disorders & 58 \\
$\quad$ (2) Bipolar disorders and schizoaffective, bipolar type & 69 \\
(3) Personality disorders & 74 \\
$\quad$ (4) Depressive and anxiety disorders & 18 \\
$\quad$ (5) Mental retardation & 12 \\
Hospitalizations ( $n$ ) & 504 \\
Number of hospitalizations during the studied period (mean \pm SD) & $1.9 \pm 1.6$ \\
Actual comorbidity with a substance abuse or dependence $(\%)$ & $216,43 \%$ \\
Bipolar disorders and schizoaffective, bipolar type $(n, \%)$ & $137,27.2 \%$ \\
Personality disorders $(n, \%)$ & $190,37.7 \%$ \\
Psychotic disorders $(n, \%)$ & $113,22.4 \%$ \\
Depressive and anxiety disorders $(n, \%)$ & $29(5.8 \%)$ \\
Mental retardation $(n, \%)$ & $35(6.9 \%)$ \\
Duration in days (mean \pm SD; median) & $19.3 \pm 22.8 ; 10$ \\
Civil compulsory hospitalization $(\%)$ & $22 \%$ \\
Quetiapine discharge dosage in mg/day (mean \pm SD; median) & $427.6 \pm 316.2 ; 400$ \\
Doses $\geq 800$ mg/day at discharge $(n, \%)$ & $73(14.5 \%)$ \\
\hline
\end{tabular}


Table 2 Odds ratio to receive quetiapine at dosage $\geq 800 \mathrm{mg} / \mathrm{day}$ at discharge (for diagnostic variable, psychotic disorders is the reference group)

\begin{tabular}{llll}
\hline Variable & OR & $95 \%$ CI & $P$-value \\
\hline Age & 0.96 & $0.93-0.98$ & $<0.0005$ \\
Benzodiazépine dosage & 1.07 & $0.98-1.16$ & 0.1 \\
Substance abuse/dependence & 0.6 & $0.2-1.3$ & 0.2 \\
Gender & 1.003 & $0.44-2.27$ & 0.9 \\
Diagnostic & - & - & 0.002 \\
$\quad$ Psychotic disorders & 1 & - & - \\
$\quad$ Bipolar disorder spectrum & 2.3 & $0.9-5.8$ & 0.09 \\
$\quad$ Unipolar depression and anxiety disorders & 0.3 & $0.03-2.7$ & 0.3 \\
$\quad$ Personality disorders & 0.1 & $0.03-0.6$ & 0.007 \\
$\quad$ Mental retardation & 0.3 & $0.03-2.7$ & 0.3 \\
\hline
\end{tabular}

\section{Discussion}

In accordance with previous naturalistic studies [5], it seems that quetiapine is given commonly in off-label as well as in labeled indication for the treatment of a large range of psychiatric disorders. The main finding of this study is that the use of quetiapine discharge doses ( $<800$ vs. $\geq 800 \mathrm{mg} /$ day) is clearly influenced by diagnostic categories whereas benzodiazepine discharge dosage, co-morbid substance abuse or dependence did not contribute significantly to the final model. Personality disorders are less likely than psychotic disorders to need quetiapine dosage at discharge higher than $800 \mathrm{mg} / \mathrm{day}$. Clinical differences across diagnostic categories could explain the finding, however one could hypothesize that clinicians are more likely to dose above licensed doses for licensed indications but not for unlicensed indications. Interestingly, the study found a link between age and quetiapine discharge doses. This may be due to differences of symptomatology between younger and older people or to a poorer tolerance of higher dosage in older patients. We excluded only 38 discharge letters of patients who were not discharged with quetiapine. Whereas all discharge letters are expected to mention all treatments used during a hospitalization, it is possible that discharge letters may have failed to mention a quetiapine administration.

Some limitations have to be considered when interpreting the results of the present study. Especially, its open-label retrospective design, lack of prospective outcome measures, and the observation limited to the discharge period.

The result suggests the need for conducting prospective randomized controlled trials (with flexible or with multiple fixed doses including dosage higher than $800 \mathrm{mg} /$ day) in order to assess efficacy and optimal doses of quetiapine in various psychiatric disorders such as unipolar depression, anxiety disorders, personality disorders, bipolar disorders and schizophrenia.

\section{Conclusion}

Quetiapine is used in a variety of clinical situations, in a wide range of doses, with a lower dosage for patients treated for a personality disorder. 


\section{References}

1. Nasrallah HA, Tandon R: Efficacy, safety, and tolerability of quetiapine in patients with schizophrenia. Journal of Clinical Psychiatry 63(Suppl 13):12-20, 2002

2. Brahm NC, Gutierres SL, Carnahan RM: Quetiapine for acute mania in bipolar disorder. American Journal of Health System Pharmacy 64(10):1045-1053, 2007. doi:10.2146/ajhp060527

3. Keating GM, Robinson DM: Quetiapine: A review of its use in the treatment of bipolar depression. Drugs 67(7):1077-1095, 2007. doi:10.2165/00003495-200767070-00008

4. Citrome L, Jaffe A, Levine J, et al.: Dosing of quetiapine in schizophrenia: How clinical practice differs from registration studies. Journal of Clinical Psychiatry 66(12):1512-1516, 2005

5. Botvinik L, Ng C, Schweitzer I: Audit of antipsychotic prescribing in a private psychiatric hospital. Australas Psychiatry 12(3):227-233, 2004. doi:10.1111/j.1039-8562.2004.02099.x

6. Adityanjee, Schulz SC: Clinical use of quetiapine in disease states other than schizophrenia. Journal of Clinical Psychiatry 63(Suppl 13):32-38, 2002

7. Mohr N, Vythilingum B, Emsley RA, et al.: Quetiapine augmentation of serotonin reuptake inhibitors in obsessive-compulsive disorder. International Clinical Psychopharmacology 17(1):37-40, 2002. doi: 10.1097/00004850-200201000-00006

8. Pinkofsky HB, Hahn AM, Campbell FA, et al.: Reduction of opioid-withdrawal symptoms with quetiapine. Journal of Clinical Psychiatry 66(10):1285-1288, 2005

9. Martinotti G, Andreoli S, Di Nicola M, et al.: Quetiapine decreases alcohol consumption, craving, and psychiatric symptoms in dually diagnosed alcoholics. Human Psychopharmacology 23(5):417-428, 2008

10. Baune BT, Caliskan S, Todder D: Effects of adjunctive antidepressant therapy with quetiapine on clinical outcome, quality of sleep and daytime motor activity in patients with treatment-resistant depression. Human Psychopharmacology 22(1):1-9, 2007. doi:10.1002/hup.817

11. Walker C, Thomas J, Allen TS: Treating impulsivity, irritability, and aggression of antisocial personality disorder with quetiapine. International Journal of Offender Therapy and Comparative Criminology 47(5):556-567, 2003. doi:10.1177/0306624X03253027

12. Villeneuve E, Lemelin S: Open-label study of atypical neuroleptic quetiapine for treatment of borderline personality disorder: Impulsivity as main target. Journal of Clinical Psychiatry 66(10): 1298-1303, 2005

13. Perrella C, Carrus D, Costa E, et al.: Quetiapine for the treatment of borderline personality disorder; an open-label study. Progress in Neuro-Psychopharmacology and Biological Psychiatry 31(1):158-163, 2007. doi:10.1016/j.pnpbp.2006.08.012

14. Barbee JG, Conrad EJ, Jamhour NJ: The effectiveness of olanzapine, risperidone, quetiapine, and ziprasidone as augmentation agents in treatment-resistant major depressive disorder. Journal of Clinical Psychiatry 65(7):975-981, 2004

15. Ahearn EP, Mussey M, Johnson C, et al.: Quetiapine as an adjunctive treatment for post-traumatic stress disorder: An 8-week open-label study. International Clinical Psychopharmacology 21(1):29-33, 2006. doi:10.1097/01.yic.0000182116.49887.ae

16. Denys D, de Geus F, van Megen HJ, et al.: A double-blind, randomized, placebo-controlled trial of quetiapine addition in patients with obsessive-compulsive disorder refractory to serotonin reuptake inhibitors. Journal of Clinical Psychiatry 65(8):1040-1048, 2004

17. Sevincok L, Topuz A: Lack of efficacy of low doses of quetiapine addition in refractory obsessivecompulsive disorder. Journal of Clinical Psychopharmacology 23(5):448-450, 2003

18. Pierre JM, Wirshing DA, Wirshing WC, et al.: High-dose quetiapine in treatment refractory schizophrenia. Schizophrenia Research 73(2-3):373-375, 2005. doi:10.1016/j.schres.2004.07.014

19. Khazaal Y, Tapparel S, Chatton A, et al.: Quetiapine dosage in bipolar disorder episodes and mixed states. Progress in Neuro-Psychopharmacology and Biological Psychiatry 31(3):727-730, 2007. doi: 10.1016/j.pnpbp.2007.01.006 